Gut and Liver, Vol. 9, No. 6, November 2015, pp. 734-740

\title{
Changes in the Expression and Distribution of Claudins, Increased Epithelial Apoptosis, and a Mannan-Binding Lectin-Associated Immune Response Lead to Barrier Dysfunction in Dextran Sodium Sulfate-Induced Rat Colitis
}

\author{
Bosi Yuan*, Shuping Zhou ${ }^{\dagger}$, Youke Lu*, Jiong Liu*, Xinxin Jin*, Haijun Wan*, and Fangyu Wang* \\ ${ }^{*}$ Department of Gastroenterology and Hepatology, Jinling Hospital, Nanjing University School of Medicine, Nanjing, and ${ }^{\dagger}$ Department of \\ Gastroenterology and Hepatology, The First People's Hospital of Huainan, Huainan, China
}

See editorial on page 699.

Background/Aims: This animal study aimed to define the underlying cellular mechanisms of intestinal barrier dysfunction. Methods: Rats were fed $4 \%$ with dextran sodium sulfate (DSS) to induce experimental colitis. We analyzed the sugars in 24-hour urine output by high pressure liquid chromatography. The expression of claudins, mannan-binding lectin (MBL), and MBL-associated serine proteases 2 (MASP-2) were detected in the colonic mucosa by immunohistochemistry; and apoptotic cells in the colonic epithelium were detected by the terminal deoxynucleotidyl transferase-mediated deoxyuridine triphosphate nick-end labeling method assay. Results: The lactulose and sucralose excretion levels in the urine of rats with DSS-induced colitis were significantly higher than those in the control rats. Mannitol excretion was lower and lactulose/mannitol ratios and sucralose/mannitol ratios were significantly increased compared with those in the control group ( $p<0.05$ ). Compared with the controls, the expression of sealing claudins (claudin 3 , claudin 5 , and claudin 8 ) was significantly decreased, but that of claudin 1 was increased. The expression of pore-forming claudin 2 was upregulated and claudin 7 was downregulated in DSS-induced colitis. The epithelial apoptotic ratio was $2.8 \% \pm 1.2 \%$ in controls and was significantly increased to $7.2 \% \pm 1.2 \%$ in DSS-induced colitis. The expression of MBL and MASP-2 in the intestinal mucosa showed intense staining in controls, whereas there was weak staining in the rats with colitis. Conclusions: There was increased intestinal permeability in DSS-induced colitis. Changes in the expression and distribution of claudins, increased epithelial apoptosis, and the MASP-2-induced immune response impaired the intestinal epithelium and contributed to high intestinal permeability. (Gut Liver 2015;9:734-740)

Key Words: Colitis, ulcerative; Claudin; Intestinal permeability; Apoptosis; Mannan-binding lectin-associated serine proteases 2

\section{INTRODUCTION}

Ulcerative colitis (UC) is a phenotype of inflammatory bowel disease (IBD) characterized by a chronic recurrent colonic inflammation, of which the clinical features are well known. This disease seems to be the consequence of an abnormal immune response to environmental factors in genetically predisposed individuals, but the etiology and exact pathophysiology are still to be fully elucidated..$^{1-3}$ Data from various studies have demonstrated that the colonic epithelium is abnormal in structure and function in patients with UC., ${ }^{4,5}$ Defective epithelial barrier function, which can contribute to disease pathogenesis or progression and be measured as increased intestinal permeability, has been implicated in $\mathrm{IBD}^{6}$ and can predict relapse during clinical remission. ${ }^{7}$ Therefore, the mechanisms of barrier function and permeability defects are thought to have a great potential in defining UC pathogenesis and guiding the development of novel treatments.

The mucosal barrier is constituted of the single layer of epithelial cells that line the intestine, which are tightly connected by a surrounding system of tight junction strands. ${ }^{8}$ Several proteins are composed of the molecular basis of tight junction strands. ${ }^{9-11}$ Although occludin and junctional adhesion molecule

Correspondence to: Fangyu Wang

Department of Gastroenterology and Hepatology, Jinling Hospital, No.305, East Zhongshan Road, Nanjing 210002, China

Tel: +86-25-8086-3384, Fax: +86-25-8086-3384, E-mail: cat409@126.com

Received on April 20, 2014. Revised on August 1, 2014. Accepted on September 11, 2014. Published online February 26,2015

pISSN 1976-2283 eISSN 2005-1212 http://dx.doi.org/10.5009/gnl14155

Bosi Yuan and Shuping Zhou contributed equally to this study as first authors.

(a) This is an Open Access article distributed under the terms of the Creative Commons Attribution Non-Commercial License (http://creativecommons.org/licenses/by-nc/4.0) which permits unrestricted non-commercial use, distribution, and reproduction in any medium, provided the original work is properly cited. 
do not predominantly involve in formation of barrier function, this is accomplished by the protein family of claudins. ${ }^{12,13} \mathrm{Sev}$ eral of at least 27 claudins members interact in a tissue-specific distribution to form a paracellular ion flux barrier. ${ }^{14}$ It has been known that the impaired epithelial barrier function in IBD. Differential expression of various members of the claudins family is the possible causes of barrier dysfunction. For example, Weber et al. ${ }^{15}$ found claudin 1 and claudin 2 expression was elevated in active IBD, adenomas, and IBD-associated dysplasia. Zeissig et al. ${ }^{16}$ found changes in expression and distribution of claudin 2, 5 , and 8 lead to altered tight junction structure and pronounced barrier dysfunction in mild to moderately active Crohn's disease. The other study by Prasad et al. ${ }^{17}$ suggest that interferon $\gamma /$ tumor necrosis factor $\alpha$ and interleukin-13 have differential effects on claudin 2, 3, and 4 in tight junctions, which may lead to increased permeability in IBD.

Beyond tight junction changes, epithelial apoptosis can also lead to barrier dysfunction. ${ }^{18}$ Thus, apoptotic epithelial cells were shown to be a potential pathogenetic factor of mucosa injury. ${ }^{19,20}$ Active UC patients have extensive mucosal tissue injury in their involved areas and apoptosis of epithelial cells may play an important role in it.

The epithelial cell barrier function is critical in providing protection against the stimulation of mucosal immune system by the intestinal microorganisms. Dysfunction of the mucosal immune system will contribute to the development of $\mathrm{IBD},{ }^{21}$ but the mechanisms of immune dysregulation in IBD are not fully defined. Mannan-binding lectin (MBL) is the main component of the lectin pathway of complement and part of the innate immune defence. It binds to the surface of microorganisms leads to the activation of MBL-associated serine proteases 2 (MASP2) and thereafter to the activation of other complement system components. $^{22,23}$ Genetic and immunohistochemistry studies suggest an involvement of MBL in the pathogenesis of $\mathrm{IBD},{ }^{24-26}$ but the exact pathophysiology is still not elucidated.

In this context, we aimed to investigate intestinal permeability in dextran sodium sulfate (DSS)-induced rat colitis by testing urinary excretion of orally administered sugars, and further investigate the changes in expression and distribution of claudins, intestinal epithelial cell apoptosis and changes in expression of MBL and MASP-2 impacted on the mechanisms of barrier dysfunction.

\section{MATERIALS AND METHODS}

\section{Animals and DSS-induced colitis model}

Three-month-old Sprague-Dawley rats were obtained from Jinling Hospital (Nanjing, China). Rats were maintained under specific pathogen-free conditions in Jinling Hospital Animal Care Facility in accordance with guidelines of Jinling Hospital Animal Care and Usage Committee. All rats were fed with autoclaved food and water and maintained in a specific-pathogen- free facility. Eighteen rats were assigned randomly to experimental colitis group $(n=12)$ and normal control group $(n=6)$. After adapting for 1 week following arrival, the rats were given drinking water containing 4\% DSS (Wako, Osaka, Japan) for 7 days until the end of the experiment on the eighth day. Body weight, rectal bleeding and stool score (stool consistency or diarrhea) were measured daily. Stool samples of rats were evaluated on a three-point scale using a test for occult blood and macroscopic evaluation ( 0 , no occult blood; 1 , test for occult blood slightly positive; 2 , test for occult blood strongly positive; and 3, bloody stool).

\section{Measurement of intestine permeability}

On eighth day after DSS treatment, the rats were moved to the metabolic cages for collecting their 24-hour urine after intragastric administration the complex solution of the triple sugars (including lactulose $120 \mathrm{mg}$, mannito $180 \mathrm{mg}$, and sucralose $60 \mathrm{mg}$ per liter). The urine was quantitatively analyzed by high pressure liquid chromatography (HPLC column: Dionex CarbonPac PA1 ion exchange column; Column temperature: $30^{\circ} \mathrm{C}$; Detector: pulsed amperometric detector integrator main electrodes: $\mathrm{Au}$; Reference electrode: $\mathrm{AgCl}$; Mobile phase: water+ $\mathrm{NaOH}$ gradient).

\section{Histology and immunohistochemical staining}

After the rats underwent 24-hour urine collection, they were sacrificed and colons were excised. All samples were snap frozen in liquid nitrogen and stored at $-80^{\circ} \mathrm{C}$ for further analysis. Colonic morphology was examined by hematoxylin and eosin staining. Immunohistochemistry was performed using polyclonal rabbit antibodies raised against claudin 1, 2, 3, 5, 7, and 8 (Zymed, San Francisco, CA, USA), and against MBL and MASP-2 (Santa Cruz Biotechnology, Santa Cruz, CA, USA). Secondary antibodies were goat anti-rabbit IgG (Molecular Probes, Eugene, OR, USA).

\section{TUNEL assay}

The terminal deoxynucleotidyl transferase-mediated deoxyuridine triphosphate nick-end labeling method (TUNEL) assay using paraffin-embedded tissues was performed according to the manufacturer's instructions. Frozen tissues were incubated with anti-PGP9.5 or anti-CGRP, followed by fluorescent detection and 4', 6-diamidino-2-phenylindole (DAPI) counterstain. For the epithelial areas, 10 random fields were evaluated per slide.

\section{Statistical analysis}

Statistical analysis was performed with SPSS 19.0 software (IBM Corp., Armonk, NY, USA). Data obtained were presented as means \pm standard error. Paired samples were evaluated using Student t-test. A p-value of $<0.05$ was considered statistically significant. 


\section{RESULTS}

\section{General observations in DSS-induced colitis}

Compared with control group, induction of colitis resulted in marked changes in Disease Activity Index (DAI), body weight, appearance of fecal blood and general status. On day 1, colitis rats showed no obvious abnormalities on general status and fecal occult blood was negative; day 2, rats appeared lazy, messy hair, loose stools and fecal occult blood was weakly positive; day 3, rats defecated loose stools and fecal occult blood test showed strong positive; day 4, rats had loose stools and gross bloody stools; day 5, rats had positive for fecal occult blood and silghtly blood, mucus or pus in stool; day 6 and 7, rats had significantly blood, mucus or pus in stool. Compare with control group, the percentage of body weight of rats in DSSinduced colitis group was significantly increased $(0.349 \pm 0.071$ vs $0.107 \pm 0.09, \mathrm{p}<0.01)$. The DAI was gradually increased in DSS-induced colitis group.

\section{Histopathological evaluation of colitis}

After oral DSS administration for 7 days, the histology of the colon at this time was characterized by edema, crypt loss and inflammatory cell infiltration (including neutrophils and mononuclear cells) in all parts of the colon compared with control group (Fig. 1).

\section{Increased intestine permeability in DSS-induced colitis}

The sugar absorption tests is based on the oral administration of triple sugars (including lactulose, mannitol, and sucralose) that differentially cross the impaired intestinal barrier to the circulation, after which they are rapidly cleared into urine. ${ }^{27}$ Then, these probes cross the impaired intestinal barrier to the circulation and are detectable in urine after renal excretion. In this study, amount of excreted laculose in the 24-hour urine of the DSS-induced colitis group was significantly higher than that of the control group. The amount of excreted mannitol was lower in the DSS-induced colitis group. But, the total sucralose excretion and sucralose/mannitol ratio (S/M) were significantly higher in the DSS-induced colitis group when compared to those of the control group (Table 1).

\section{Changes in expression and distribution of claudins in DSS-induced colitis}

In the DSS-induced colitis group, a weak expression of claudin 1 was detected in colonic epithelial cells, whereas it was absent in the control group. Claudin 2 and 3 were well expressed on the top of colonic crypts and lateral epithelium in the control group, whereas in the DSS-induced colitis group, claudin 2

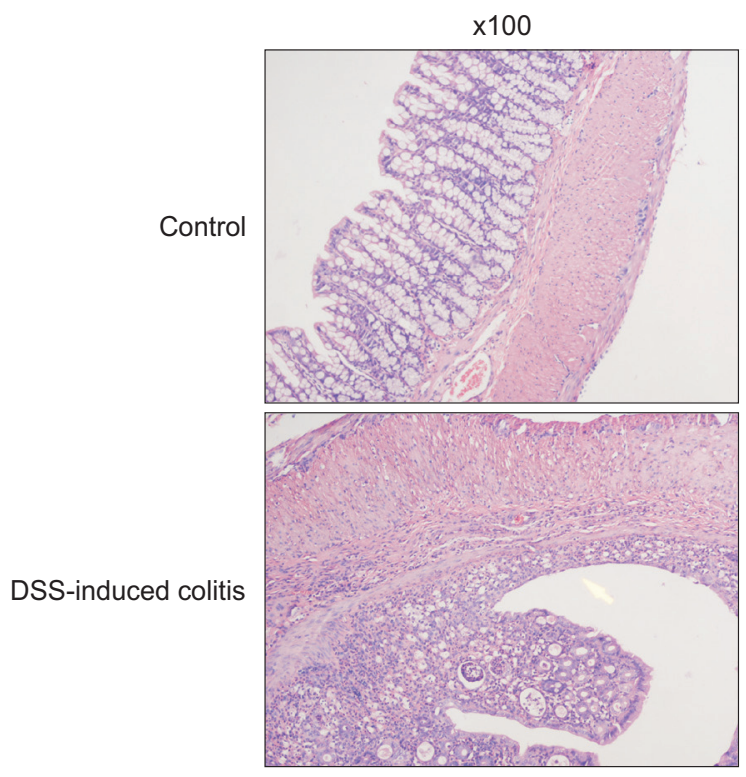

$x 100$

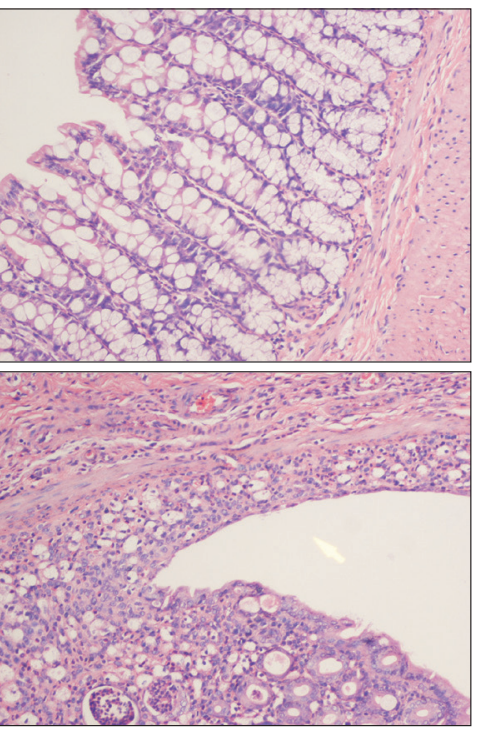

Fig. 1. Edema, crypt loss, and inflammatory cell infiltration of the colon were found in the dextran sodium sulfate (DSS)-induced colitis group compared with the control group (magnifications: left, $\times 100$; right, $\times 200)$.

Table 1. Triple Sugar Excretion in the 24-Hour Urine of Rats

\begin{tabular}{lccccc}
\hline \multicolumn{1}{c}{ Group } & Mannitol, mg/L & Lactulose, $\mathrm{mg} / \mathrm{L}$ & Sucralose, $\mathrm{mg} / \mathrm{L}$ & L/M & S/M \\
\hline Control & $0.0185 \pm 0.00224$ & $0.0546 \pm 0.0035$ & $0.1228 \pm 0.095$ & $3.8 \pm 2.3$ & $8.4 \pm 6.4$ \\
DSS-induced colitis & $0.0155 \pm 0.00218$ & $1.6289 \pm 0.3273$ & $0.6921 \pm 0.1348$ & $107.5 \pm 29.2$ & $45.1 \pm 9.6$ \\
p-value* & 0.017 & $<0.001$ & $<0.001$ & $<0.001$ & $<0.001$ \\
\hline
\end{tabular}

Data are presented as mean \pm SD.

L/M, lactulose/mannitol; S/M, sucralose/mannitol; DSS, dextran sodium sulfate.

*Control versus DSS-induced colitis. 


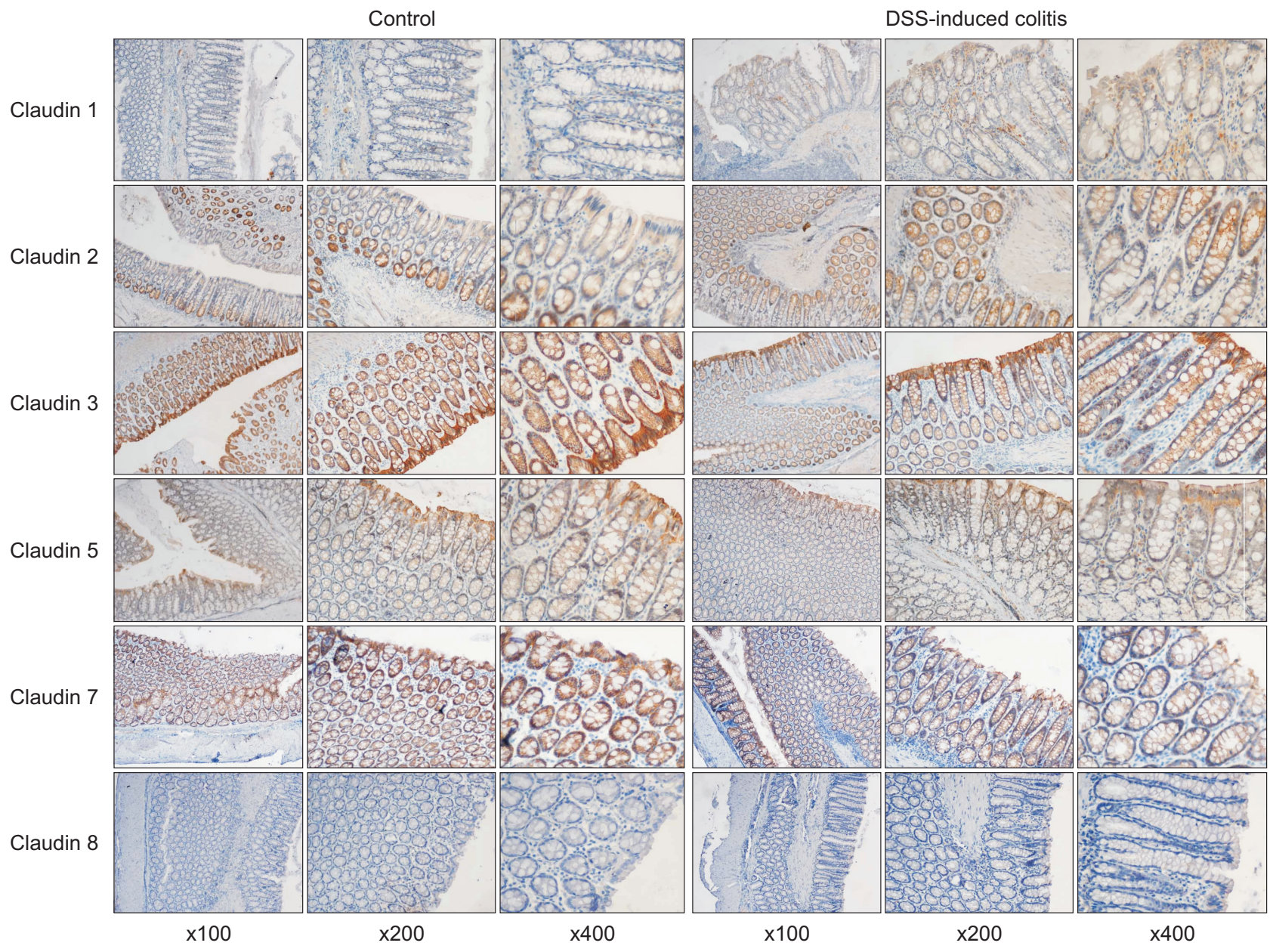

Fig. 2. The expression of claudin 1 was absent in the control group, and there was faint labeling in the epithelial cells lining the crypts in the dextran sodium sulfate (DSS)-induced colitis group. Strong claudin 2 labeling was visible from the bottom of the crypts to the surface of the colonic mucosa in the DSS-induced colitis group; the gradient of expression visible in the controls was partly preserved. Strong expression of claudin 3 was observed in the colonic crypts and epithelium in both groups, but the expression levels were lower in the colonic mucosa of the colitis group. Claudin 5 and claudin 7 exhibited predominant expression on the top of the colonic crypts and at the base of the colonic epithelial cells in the control group, whereas there was decreased staining in the colitis group. Claudin 8 showed weak staining in the control group and absent expression in the colitis group (magnifications: left, $\times 100$; middle, $\times 200$; right, $\times 400$ ).

showed a stronger expression, claudin 3 showed a decreased staining. Claudin 5 and 7 exhibited a predominantly expression on the top of colonic crypts and the base of colonic epithelial cells of the control group, whereas they were showed decreased staining in the DSS-induced colitis group. Claudin 8 showed weak staining in the control group and absent expression in the DSS-induced colitis group (Fig. 2).

\section{Increased intestinal epithelial apoptosis in DSS-induced colitis}

Intestinal epithelial apoptosis was investigated in TUNEL sections of colon from the control group and DSS-induced colitis group. The epithelial apoptotic ratio was $2.8 \% \pm 1.2 \%$ in the control group and was significantly increased to $7.2 \% \pm 1.2 \%$ in the
DSS-induced colitis group ( $\mathrm{p}<0.001)$ (Fig. 3).

\section{Changes in expression and distribution of $M B L$ and MASP-2 in DSS-induced colitis}

The immunohistochemical results of the expression of MBL and MASP-2 in the intestinal mucosa showed intense staining in both surface and crypt epithelium of the control group, whereas those in the DSS-induced colitis group showed weak staining (Fig. 4). The integrated optical density indicated the positive expression area and intensity of MBL and MASP-2. The integrated optical density of expression of MBL and MASP2 were $39.998 \pm 5.965$ and $18.904 \pm 1.302$ in control group, and were $21.173 \pm 0.389$ and $11.892 \pm 0.942$ in colitis group, respectively $(\mathrm{p}<0.05)$. 


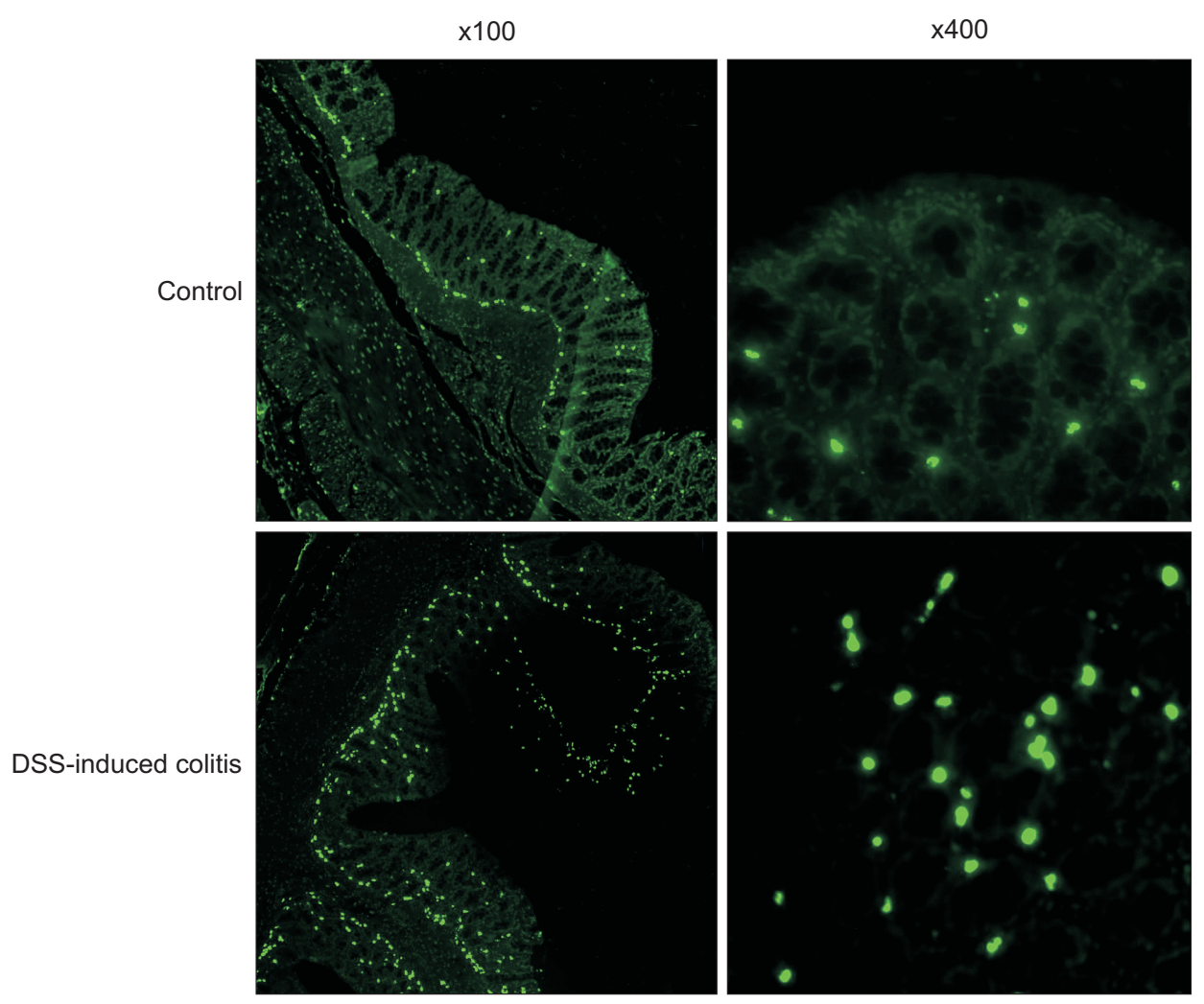

Fig. 3. Intestinal epithelial cells of rats with dextran sodium sulfate (DSS)-induced colitis contained considerably more fragmented DNA (green fluorescence) than cells on a normal diet (magnifications: left, $\times 100$; right, $\times 400$ ).

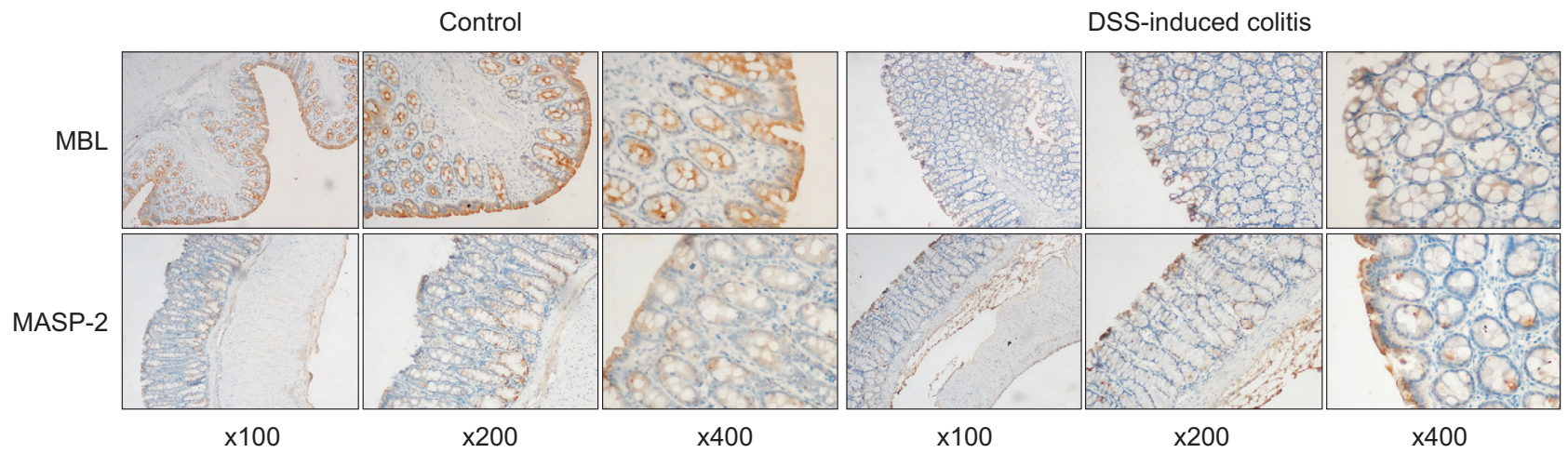

Fig. 4. The immunohistochemical results of the expression of mannan-binding lectin (MBL) and MBL-associated serine proteases 2 (MASP-2) in the intestinal mucosa showed intense staining in both surface cells and the crypt epithelium of the control group, whereas those of the colitis group showed weak staining (left, $\times 100$; middle, $\times 200$; right, $\times 400$ ).

DSS, dextran sodium sulfate.

\section{DISCUSSION}

In this study, we showed higher intestinal permeability in the DSS-induced colitis group compared with the control group. Greatly increased intestinal permeability reflects intestinal barrier dysfunction. Intestinal barrier dysfunction is a main feature of UC, which can lead to leak-flux diarrhea and a facilitated uptake of noxious antigens, but the formal pathogenesis remains to be elucidated. DSS-induced colitis is one of the most commonly used animal models of UC, it can reflect many of the clinical features of UC. ${ }^{28,29}$ In this study, we investigated mechanisms of barrier impairment in UC from tight junction changes, epithelial apoptosis and mucosal immune system.

The tissue-specific expression of claudins determines the barrier characteristics of the respective epithelium. Whereas several of the 27 claudins can either increase or decrease the expression on epithelial in different Intestinal diseases, including IBD. ${ }^{30}$ Since a little is known about the claudins in UC, we investigated distribution of some gut-specific subset of claudins which are characterized based on their effects on barrier function. In accordance with their electrophysiological properties, we found constitutive expression of sealing tight junction proteins (claudin 3, 4, 5, and 8) and the pore forming proteins (claudin 2 and 7), no expression of sealing protein claudin 1 in control groups rat 
colon. In contrast with controls, expression of sealing claudins (claudin 3, 5, and 8) which decrease cation permeability was greatly decreased in DSS-induced colitis, combining with increased expression of claudin 1 , which is believed to decrease sealing properties. ${ }^{31}$ Moreover, the pore forming claudin 2 was dramatically upregulated, accompanied with low expression of claudin 8, which indicates that claudin 2 may indeed substitute for claudin 8 to reduce the apparent number of functional cation pores. ${ }^{32}$ Besides, downregulated expression of pore forming claudin 7 was shown to increase paracellular cation permeability and thus did not contribute to barrier function. Therefore, downregulation and redistribution of sealing claudins with increased expression of the pore-forming claudin 2 and decreased claudin 7 enhance tight junction permeability for cations, thus providing the molecular basis for leakflux diarrhea in UC.

Recent findings have highlighted that intestinal epithelial cells are a pivotal physiological barrier between the environment and the host. ${ }^{33-35}$ They are linked together at the apical junctional complex by tight junctions while forming a physical barrier and crucial for coordinating the ensuing immune response. ${ }^{36,37}$ Apoptosis of intestinal epithelial cells contributes to tissue damage under different conditions including acute and chronic intestinal inflammation. In this study, we showed the apoptotic ratio differ between control group and DSS-induced colitis. As intestinal epithelial cells apoptosis had highly increased and higher intestinal permeability in DSS-induced colitis, a significant contribution of apoptosis to barrier dysfunction must be assumed for UC.

Intestinal epithelial barrier dysfunction can broadly activate mucosal immune responses and accelerate the onset and severity of immune-mediated colitis. ${ }^{38}$ Dysregulation of the mucosal immune system is a main factor contributing to the pathogenesis of IBD. ${ }^{1}$ MBL plays an important role in nonspecific immunity and complement activation. Given the importance of MBL in immune defense against microorganisms, ${ }^{39,40} \mathrm{MBL}$ could be an important factor in the protection against etiological microbial agents in IBD. In this study, we investigated the expression of the MBL and MASP-2 in DSS-induced colitis, and our results showed MBL and MASP-2 were significantly lower in colitis group when compared with controls, and those results are consistent with increased intestinal permeability, tissue injury and neutrophil infiltration in DSS-induced colitis. Clinical study also confirmed MBL levels were lower in paediatric patients with IBD than in controls. ${ }^{41}$ In animal experiment, ${ }^{42}$ gastrointestinal ischemia-reperfusion significantly increased gastrointestinal barrier dysfunction and neutrophil infiltration into the gut, MBL-null mice displayed little gut injury after gastrointestinal ischemiareperfusion. Addition of recombinant human MBL to MBL-null mice significantly increased injury compared with MBL-null mice after gastrointestinal ischemia-reperfusion and was reversed by anti-MBL antibody treatment. Although the model of UC is difference from gastrointestinal ischemia-reperfusion, they showed some identical aspects of gut injury. As MBL mutations decrease the formation of functional MBL could protect against the clinical development of sporadic UC, ${ }^{26}$ decreased expression of MBL and MASP-2 might as a protective factor against UC. Therefore we supposed MBL induced immune response impaired barrier function contributed to high intestinal permeability, further studies are required for clarify the exact mechanism of signal regulation.

Taken together, our findings provide evidence that upregulation of pore-forming claudin 2, downregulation of pore-forming claudin 7 , downregulation and redistribution of sealing claudin 3, 5, and 8 led to alter tight junction structure and pronounced barrier dysfunction in DSS-induced colitis. Apoptosis intestinal epithelial cells increased intestinal permeability and contributed to barrier dysfunction. Then barrier dysfunction activated MASP-2 induced immune response impaired intestinal epithelial barrier function. Therefore, three different mechanisms of impaired intestinal epithelial barrier function possibly common contributed to high intestinal permeability, further studies are required for clarify the exact mechanism of signal regulation.

\section{CONFLICTS OF INTEREST}

No potential conflict of interest relevant to this article was reported.

\section{ACKNOWLEDGEMENTS}

This study was supported by Research Grants from National Natural Science Foundation of China, No. 81070289.

We thank Prof. Zhang Xinhua from Jinling Hospital for pathological picture analysis.

\section{REFERENCES}

1. Xavier RJ, Podolsky DK. Unravelling the pathogenesis of inflammatory bowel disease. Nature 2007;448:427-434.

2. Strober W, Fuss I, Mannon P. The fundamental basis of inflammatory bowel disease. J Clin Invest 2007;117:514-521.

3. Colombel JF, Watson AJ, Neurath MF. The 10 remaining mysteries of inflammatory bowel disease. Gut 2008;57:429-433.

4. Esaki M, Kubokura N, Kudo T, Matsumoto T. Endoscopic findings under narrow band imaging colonoscopy in ulcerative colitis. Dig Endosc 2011;23 Suppl 1:140-142.

5. Gilchrist KW, Harms BA, Starling JR. Abnormal rectal mucosa of the anal transitional zone in ulcerative colitis. Arch Surg 1995;130:981-983.

6. Salim SY, Söderholm JD. Importance of disrupted intestinal barrier in inflammatory bowel diseases. Inflamm Bowel Dis 2011;17:362381.

7. Kiesslich R, Duckworth CA, Moussata D, et al. Local barrier dysfunction identified by confocal laser endomicroscopy predicts relapse in inflammatory bowel disease. Gut 2012;61:1146-1153.

8. Schneeberger EE, Lynch RD. The tight junction: a multifunctional 
complex. Am J Physiol Cell Physiol 2004;286:C1213-C1228.

9. Furuse M, Hirase T, Itoh M, et al. Occludin: a novel integral membrane protein localizing at tight junctions. J Cell Biol 1993;123(6 Pt 2):1777-1788.

10. Furuse M, Sasaki H, Fujimoto K, Tsukita S. A single gene product, claudin-1 or -2 , reconstitutes tight junction strands and recruits occludin in fibroblasts. J Cell Biol 1998;143:391-401.

11. Tsukita S, Furuse M, Itoh M. Multifunctional strands in tight junctions. Nat Rev Mol Cell Biol 2001;2:285-293.

12. Turksen K, Troy TC. Barriers built on claudins. J Cell Sci 2004; 117(Pt 12):2435-2447.

13. Mandell KJ, Parkos CA. The JAM family of proteins. Adv Drug Deliv Rev 2005;57:857-867.

14. Hartsock A, Nelson WJ. Adherens and tight junctions: structure, function and connections to the actin cytoskeleton. Biochim Biophys Acta 2008;1778:660-669.

15. Weber CR, Nalle SC, Tretiakova M, Rubin DT, Turner JR. Claudin-1 and claudin-2 expression is elevated in inflammatory bowel disease and may contribute to early neoplastic transformation. Lab Invest 2008;88:1110-1120.

16. Zeissig S, Bürgel N, Günzel D, et al. Changes in expression and distribution of claudin 2, 5 and 8 lead to discontinuous tight junctions and barrier dysfunction in active Crohn's disease. Gut 2007; 56:61-72.

17. Prasad S, Mingrino R, Kaukinen K, et al. Inflammatory processes have differential effects on claudins 2, 3 and 4 in colonic epithelial cells. Lab Invest 2005;85:1139-1162.

18. Nielsen HL, Nielsen H, Ejlertsen T, et al. Oral and fecal Campylobacter concisus strains perturb barrier function by apoptosis induction in HT-29/B6 intestinal epithelial cells. PLoS One 2011;6: e23858.

19. Goretsky T, Dirisina R, Sinh P, et al. p53 mediates TNF-induced epithelial cell apoptosis in IBD. Am J Pathol 2012;181:1306-1315.

20. Liapis G, Boletis J, Skalioti C, et al. Histological spectrum of mycophenolate mofetil-related colitis: association with apoptosis. Histopathology 2013;63:649-658.

21. Cader MZ, Kaser A. Recent advances in inflammatory bowel disease: mucosal immune cells in intestinal inflammation. Gut 2013; 62:1653-1664.

22. Degn SE, Jensen L, Hansen AG, et al. Mannan-binding lectinassociated serine protease (MASP)-1 is crucial for lectin pathway activation in human serum, whereas neither MASP-1 nor MASP-3 is required for alternative pathway function. J Immunol 2012;189: 3957-3969.

23. Héja D, Kocsis A, Dobó J, et al. Revised mechanism of complement lectin-pathway activation revealing the role of serine protease MASP-1 as the exclusive activator of MASP-2. Proc Natl Acad Sci U S A 2012;109:10498-10503.

24. Boniotto M, Braida L, Spanò A, et al. Variant mannose-binding lectin alleles are associated with celiac disease. Immunogenetics 2002;54:596-598.

25. Iltanen S, Mäki M, Collin P, et al. The association between mannan-binding lectin gene alleles and celiac disease. Am J Gastroen- terol 2003:98:2808-2809.

26. Rector A, Lemey P, Laffut W, et al. Mannan-binding lectin (MBL) gene polymorphisms in ulcerative colitis and Crohn's disease. Genes Immun 2001;2:323-328.

27. Grootjans J, Thuijls G, Verdam F, Derikx JP, Lenaerts K, Buurman WA. Non-invasive assessment of barrier integrity and function of the human gut. World J Gastrointest Surg 2010;2:61-69.

28. Yan Y, Kolachala V, Dalmasso G, et al. Temporal and spatial analysis of clinical and molecular parameters in dextran sodium sulfate induced colitis. PLoS One 2009;4:e6073.

29. Perše M, Cerar A. Dextran sodium sulphate colitis mouse model: traps and tricks. J Biomed Biotechnol 2012;2012:718617.

30. Krause G, Winkler L, Mueller SL, Haseloff RF, Piontek J, Blasig IE. Structure and function of claudins. Biochim Biophys Acta 2008; 1778:631-645.

31. Sonoda N, Furuse M, Sasaki H, et al. Clostridium perfringens enterotoxin fragment removes specific claudins from tight junction strands: evidence for direct involvement of claudins in tight junction barrier. J Cell Biol 1999;147:195-204.

32. Yu AS, Enck AH, Lencer WI, Schneeberger EE. Claudin-8 expression in Madin-Darby canine kidney cells augments the paracellular barrier to cation permeation. J Biol Chem 2003;278:1735017359.

33. Peterson LW, Artis D. Intestinal epithelial cells: regulators of barrier function and immune homeostasis. Nat Rev Immunol 2014;14:141-153.

34. Turner JR. Intestinal mucosal barrier function in health and disease. Nat Rev Immunol 2009;9:799-809.

35. Kagnoff MF. The intestinal epithelium is an integral component of a communications network. J Clin Invest 2014;124:2841-2843.

36. Rescigno M. The intestinal epithelial barrier in the control of homeostasis and immunity. Trends Immunol 2011;32:256-264.

37. Catalioto RM, Maggi CA, Giuliani S. Intestinal epithelial barrier dysfunction in disease and possible therapeutical interventions. Curr Med Chem 2011;18:398-426.

38. Su L, Shen L, Clayburgh DR, et al. Targeted epithelial tight junction dysfunction causes immune activation and contributes to development of experimental colitis. Gastroenterology 2009;136: 551-563.

39. Turner MW. Mannose-binding lectin (MBL) in health and disease. Immunobiology 1998;199:327-339.

40. Garred P, Madsen HO, Hofmann B, Svejgaard A. Increased frequency of homozygosity of abnormal mannan-binding-protein alleles in patients with suspected immunodeficiency. Lancet 1995; 346:941-943.

41. Kovacs M, Papp M, Lakatos PL, et al. Low mannose-binding lectin (MBL) is associated with paediatric inflammatory bowel diseases and ileal involvement in patients with Crohn disease. J Crohns Colitis 2013;7:134-141.

42. Hart ML, Ceonzo KA, Shaffer LA, et al. Gastrointestinal ischemiareperfusion injury is lectin complement pathway dependent without involving C1q. J Immunol 2005;174:6373-6380. 